\title{
The Scarborough express train crash: four case reports
}

\author{
P. A. STONEBRIDGE AND P. E. RANDALL
}

Hope Hospital, Salford, England

\section{SUMMARY}

Four cases are presented of patients who sustained blunt trauma to the epigastrium in a train crash. Two patients sustained a transected pancreas. The mechanism of injury is discussed.

\section{INTRODUCTION}

At 10.55 am on 4 December 1984 the Liverpool-Scarborough express collided with the rear of a stationary fuel train in Salford. The driver was killed and fire engulfed the first two carriages. Fortunately, no passengers were in that part of the train. Seventy-eight passengers sustained minor injury, and only four people required admission.

These four cases are presented.

\section{CASE REPORTS}

\section{Case 1}

A 63-year-old woman developed severe upper abdominal pain which gradually diminished over 1 hour, returning with increased severity 4 hours later. She was not shocked but was tender in the epigastrium, with no rebound and normal bowel sounds. Serum, amylase, chest and abdominal radiographs, and ECG were all normal. She was observed overnight and took her own discharge the following day.

Correspondence: $\operatorname{Dr}$ P. A. Stonebridge, Department of Surgery, Hope Hospital, Eccles Old Road, Salford 6, England 
Case 2

A 20-year-old girl complained of severe epigastric pain, associated with nausea and retching. On examination, blood pressure and pulse were normal, she had a tender epigastrium with no rebound and normal bowel sounds. All investigations were normal. She was observed overnight and discharged the following day as her symptoms had $\stackrel{\overline{\vec{s}}}{\stackrel{\vec{m}}{ }}$ settled.

Case 3

A 72-year-old man complained of epigastric pain, he was not shocked at the scene of the accident, but was on arrival at hospital 10 minutes later (BP 70/50 $\mathrm{mmHg}$ ). He had a rigid abdomen and absent bowel sounds. At laparotomy he was found to have $\vec{\omega}$ transection of the pancreas to the right of the neck with avulsion of the superior $\underset{\mathbb{D}}{\mathbb{N}}$ mesenteric vein from the portal vein, and transection of the common bile duct and the 3 . right hepatic artery. Despite attempts to treat the injuries by a modified Whipple's operation the patient died on the table.

\section{Case 4}

A 57-year-old man complained of severe epigastric pain. On examination, blood $\frac{c}{\sigma}$ pressure and pulse were normal; he had a bruise in the epigastrium with guarding and $\vec{\varphi}$ absent bowel sounds. At laparotomy he was found to have a tear of the transverse mesocolon, transection of the pancreatic head and a small tear of the liver capsule. subtotal pancreatectomy and splenectomy was performed. He initially recovered, but weeks later required a vagotomy and pyloroplasty for bleeding acute gastric erosions, $\frac{\partial}{0}$ despite prophylactic Cimetidine therapy. Unfortunately the patient developed renal failure and died a month after the initial incident following massive bleeding from multiple gastric erosions.

\section{DISCUSSION}

All four patients were sitting at tables facing the engine. Three were in the buffet car and one in an ordinary carriage. All patients sustained the same external injury, i.e. forced flexion over the edge of a table.

Blunt trauma is known to cause transections of the pancreas (Northrup \& Simmons, $\rightarrow$ 1972; Davis et al., 1976; Henavejos et al., 1983). These cases are of interest in that the mechanism of injury is the same (two patients sustaining a transected pancreas when $N$ forced over the tables). The train was travelling slowly (approximately $30 \mathrm{mph}$ ), having $\mathrm{N}$ just left a station, when it was brought to an abrupt halt. It is interesting that a low $\mathrm{N}_{\mathrm{\omega}}$ velocity impact created enough force to deliver sufficient blunt injury to the abdomen to 0 transect a pancreas. We must therefore conclude that the height and thickness of theco tabletops in the train was an important factor in the pathology. 


\section{ACKNOWLEDGEMENTS}

We would like to thank Mr J. R. N. Curt and Dr G. S. Laing for allowing us to present these cases.

\section{REFERENCES}

Davis J. J., Cohn I. \& Nance F. C. (1976) Diagnosis and management of blunt adominal trauma. Annals of Surgery 183, 672-7.

Henavejos A., Cohen D. M. \& Moossa A. R. (1983) Management of pancreatic trauma. Annals of the Royal College of Surgeons of England 65, 297-300.

Northrup W. F. \& Simmons R. L. (1972) Pancreatic trauma. Surgery 71, $27-43$.

Received 26 April 1985; accepted 9 May 1985 